(C) 2018. This manuscript version is made available under the CC-BY-NC-ND 4.0 license http://creativecommons.org/licenses/by-nc-nd/4.0/

\title{
Application of Two Different Methods for Extending Lattice-Valued Restricted Equivalence Functions used for Constructing Similarity Measures on L-Fuzzy Sets
}

\author{
Eduardo S. Palmeira ${ }^{\mathrm{a}}$, Benjamin Bedregal ${ }^{\mathrm{b}}$, Humberto Bustince ${ }^{\mathrm{c}, \mathrm{d}}$, Daniel \\ Paternain $^{\mathrm{c}, \mathrm{d}}$, Laura De Miguel ${ }^{\mathrm{c}, \mathrm{d}}$ \\ ${ }^{a}$ Departamento de Ciências Exatas e Tecnológicas \\ Universidade Estadual de Santa Cruz \\ Ilhéus, Brazil \\ Email: espalmeira@uesc.br \\ ${ }^{b}$ Departamento de Informática e Matemática Aplicada \\ Universidade Federal do Rio Grande do Norte \\ Natal, Brazil \\ Email: bedregal@dimap.ufrn.br \\ ${ }^{c}$ Departamento de Automatica y Computacion \\ Universidad Publica de Navarra \\ Pamplona, Spain \\ Email: \{bustince,daniel.paternain,laura.demiguel\}@unavarra.es \\ ${ }^{d}$ Institute of Smart Cities \\ Universidad Publica de Navarra
}

\begin{abstract}
Based on previous investigations, we have proposed two different methods to extend lattice-valued fuzzy connectives (t-norms, t-conorms, negations and implications) and other related operators, considering a generalized notion of sublattices. Taking into account the results obtained and seeking to analyze the behavior of both extension methods in face of fuzzy operators related to image processing, we have applied these methods so as to extend restricted equivalence functions, restricted dissimilarity functions and $E_{e, N}$-normal functions. We also generalize the concepts of similarity measure, distance measure and entropy measure for $L$-fuzzy sets constructing them via restricted equivalence functions, restricted dissimilarity functions and $E_{e, N}$-normal functions.
\end{abstract}

Keywords: Fuzzy logic, restricted equivalence functions, retractions, extension, e-operators 


\section{Introduction}

Let's consider the following issue: assuming that $M$ is a sublattice of $L$ and that $T$ is a fuzzy operator (a t-norm, a t-conorm, fuzzy negation, fuzzy implication, etc.) defined on $M$; how can we extend $T$ to $L$ preserving its properties? Our studies in recent years on this issue led us to provide two different extension methods based on a relaxed notion of sub-lattice. In [27], we presented an extension method via retractions (shortly EMR) that aimed to obtain a minimum extension of a given operator and was able to preserve its properties; yet, results have shown that some properties of fuzzy connectives are not preserved by this method. In order to fix this weakness, we have proposed in [30] another extension method that uses a special function named e-operator. Unlike EMR, the extension method via e-operators (shortly EMEP) is able to preserve most of the properties of extended operators. However, the operator generated from the extension is not minimal, in general.

A crucial step of several applications is the construction of measures for the global comparison of objects which are represented by fuzzy sets. However, there are many situations in which, due to the high degree of uncertainty, the objetcs we need to compare are better represented by extensions of fuzzy sets, such as interval-valued fuzzy sets, type-2 fuzzy sets or $L$-fuzzy sets in a more general way [9]. In these sets, membership degrees are not given by a real number in $[0,1]$, but by an element of a bounded lattice. Therefore, this kind of measures must be generalized to an arbitrary bounded lattice, which is not a trivial task.

A frequent methodology to generate these measures defined over fuzzy sets consists on the aggregation of functions which take values on $[0,1]$ (representing the membership degree). For example, those given in [11, 12] for the global comparison of grayscale images aggregates the values of restricted equivalence functions.

From our point of view, this means that the problem can be divided into two different steps:

- to develop a theoretical study of the functions used to compare the different elements of the object (for example, the elements belonging to an $L$-fuzzy set); 
- to develop a theoretical study to aggregate the local information obtained in the previous step in order to achieve a global comparison.

Therefore, the objetive of this paper is, firstly, to study the extension of some functions that are commonly used for constructing comparison measures, such as restricted equivalence functions, restricted dissimilarity functions and $E_{e, N}$-normal functions. In order to do this, we study the application of EMR and EMEP methods to extend these functions from $[0,1]$ to bounded lattices. Secondly, to study the aggregation of previous functions in order to obtain global comparison measures, namely similarity measures, distances and entropy measures to compare $L$-fuzzy sets.

We start by recalling elementary concepts in lattice theory and defining other essential ones in Section 2. In Section 3 we state some results about negations obtained from REF, whereas Section 4 is devoted to address the extension of restricted equivalence functions. In the framework we also make the extension of restricted dissimilarity functions and $E_{e, N}$-normal functions in Sections 5 and 6 respectively. The construction of similarity, dissimilarity and entropy measure is added in Section 7. Conclusion and some remarks are discussed in Section 8.

\section{Preliminaries}

\subsection{Bounded Lattices and its Morphisms}

Most definitions and results in this subsection are well known from the literature, so we just present here a clear formalization of them. Moreover, some other key concepts such as retract are defined. For a deeper reading about these concepts we recommend $[6,13,14,16,17,18,27]$.

Definition 2.1. Let $L$ be a nonempty set. If $\wedge_{L}$ and $\vee_{L}$ are two binary operations on $L$, then $\left\langle L, \wedge_{L}, \vee_{L}\right\rangle$ is a bounded lattice provided that for each $x, y, z \in L$, the following properties hold:

1. $x \wedge_{L} y=y \wedge_{L} x$ and $x \vee_{L} y=y \vee_{L} x$ (symmetry);

2. $\left(x \wedge_{L} y\right) \wedge_{L} z=x \wedge_{L}\left(y \wedge_{L} z\right)$ and $\left(x \vee_{L} y\right) \vee_{L} z=x \vee_{L}\left(y \vee_{L} z\right)$ (associativity);

3. $x \wedge_{L}\left(x \vee_{L} y\right)=x$ and $x \vee_{L}\left(x \wedge_{L} y\right)=x$ (absorption law);

4. There are elements $0_{L}$ (bottom) and $1_{L}$ (top) of $L$ such that $x \vee_{L} 0_{L}=x$ and $x \wedge_{L} 1_{L}=x$, for each $x \in L$. 
It is also possible to establish a partial order on $L$ by relation

$$
x \leqslant_{L} y \text { if and only if } x \wedge_{L} y=x
$$

Moreover, $L$ is called complete whenever each subset of $L$ has a supremum $^{1}$ and an infimum element.

Throughout this paper we take $L$ as a bounded lattice as defined above. If $L$ represents another thing, the appropriate distinction will be made.

When there are two elements $x$ and $y$ belonging to $L$ such that neither $x \leqslant_{L} y$ nor $y \leqslant_{L} x$, these elements are said to be incomparable and we denote this by $x \| y$. Otherwise we say that these elements are comparable (notation: $x \supset y$ ).

Definition 2.2. Let $\left(L, \wedge_{L}, \vee_{L}, 0_{L}, 1_{L}\right)$ and $\left(M, \wedge_{M}, \vee_{M}, 0_{M}, 1_{M}\right)$ be bounded lattices. A mapping $f: L \longrightarrow M$ is said to be a homomorphism if, for all $x, y \in L$, we have

1. $f\left(x \wedge_{L} y\right)=f(x) \wedge_{M} f(y)$;

2. $f\left(x \vee_{L} y\right)=f(x) \vee_{M} f(y)$;

3. $f\left(0_{L}\right)=0_{M}$ and $f\left(1_{L}\right)=1_{M}$.

Notice that every lattice homomorphism preserves the order (see for example [30]).

Definition 2.3. [4] Let $L$ be a bounded lattice and $L^{n}$ the Cartesian product of $n$ copies of $L$. Given a function $f: L^{n} \rightarrow L$, the action of an automorphism ${ }^{2} \rho$ of $L$ over $f$ results in the function $f^{\rho}: L^{n} \rightarrow L$ defined as

$$
f^{\rho}\left(x_{1}, \ldots, x_{n}\right)=\rho^{-1}\left(f\left(\rho\left(x_{1}\right), \ldots, \rho\left(x_{n}\right)\right)\right)
$$

In this case, $f^{\rho}$ is said to be a conjugate of $f$.

Classically, a (ordinary) sublattice of a lattice $L$ is a subset $M$ of $L$ such that $x, y \in M$ imply $x \wedge_{L} y \in M$ and $x \vee_{L} y \in M$. Here we consider a generalized notion of sublattice as in Definition 2.4 using retration as follows.

\footnotetext{
${ }^{1}$ Let $X$ be a subset of $L$. An element $a \in L(b \in L)$ is called a supremum (infimum) of $X$ if for all $x \in X, x \leqslant_{L} a\left(b \leqslant_{L} x\right)$ and if there is $a^{\prime} \in L\left(b^{\prime} \in L\right)$ such that $x \leqslant_{L} a^{\prime}$ $\left(b^{\prime} \leqslant_{L} x\right)$ then $a \leqslant_{L} a^{\prime}\left(b^{\prime} \leqslant_{L} b\right)$.

${ }^{2}$ An automorphism on $L$ is a homorphism from $L$ to $L$.
} 
Definition 2.4. [14] A homomorphism $r$ of a lattice $L$ onto a lattice $M$ is said to be a retraction if there exists a homomorphism $s$ of $M$ into $L$ which satisfies $r \circ s=i d_{M}$. A lattice $M$ is called a $(r, s)$-retract ${ }^{3}$ (or just a retract) of a lattice $L$ if there is a retraction $r$, of $L$ onto $M$, and $s$ is then called a pseudo-inverse of $r$.

Notice that saying that $M$ is a retract of $L$ means that it is possible to identify $M$ with the subset $K=s(M)$ of $L$ preserving the lattice structure of $M$ and therefore $K$ is a sublattice of $L$. In other words, $K$ is isomorphic (since $\left.r\right|_{K}$ is an isomorphism) to $M$ and hence $K$ is an algebraic copy of $M$ embedded into $L$.

Remark 1. It follows from the above definition that every retraction $r$ satifies $r\left(0_{L}\right)=0_{M}$ and $r\left(1_{L}\right)=1_{M}$. Indeed, for every $y \in M$ there is $x \in L$ such that $r(x)=y$ since $r$ is surjective. Thus $r\left(0_{L}\right)=r\left(0_{L} \wedge_{L} x\right)=r\left(0_{L}\right) \wedge_{M} r(x)=$ $r\left(0_{L}\right) \wedge_{M} y$ and hence $r\left(0_{L}\right)=0_{M}$. Similarly, one can prove that $r\left(1_{L}\right)=1_{M}$.

Definition 2.5. Every retraction $r: L \longrightarrow M$ (with pseudo-inverse $s$ ) that satisfies $s \circ r \leqslant i d_{L}{ }^{4}\left(i d_{L} \leqslant s \circ r\right)$ is called a lower (an upper) retraction. In this case, $M$ is a lower (an upper) retract of $L$.

Remark 2. It should be noted that given a lower retraction $r$ with pseudoinverse $s$, it is sometimes possible, to define an upper retraction $r^{\prime}$ that also has s as a pseudo-inverse (see Example 2.2 and Remark 2.3 in [30]). Moreover, if $M$ is a retract of $L$, then the retraction $r$ could be lower, upper or neither.

Definition 2.6. Let $M$ be a $\left(r_{1}, s\right)$-retract of $L$. We say that

1. $M$ is a lower $\left(r_{1}, s\right)$-retract of $L$ if $r_{1}$ is a lower retraction. Notation: $M<L$ with respect to $\left(r_{1}, s\right)$;

2. $M$ is an upper $\left(r_{1}, s\right)$-retract of $L$ whenever $r_{1}$ is an upper retraction. Notation: $M>L$ with respect to $\left(r_{1}, s\right)$;

\footnotetext{
${ }^{3}$ In papers $[27,28,30,29]$ we call $(r, s)$-retract by $(r, s)$-sublattice in order to emphazise that for each pair of homormorphims $(r, s)$ we have different retracts.

${ }^{4}$ Let $f$ and $g$ be functions on a lattice $L$. We write $f \leqslant g$ when $f(x) \leqslant L g(x)$ for all $x \in L$.
} 
3. If $r_{1}$ is a lower retraction and there is an upper retraction $r_{2}: L \longrightarrow M$ such that its pseudo-inverse is also $s$, then $M$ is called a full $\left(r_{1}, r_{2}, s\right)$ retract of $L$. Notation: $M \unlhd L$ with respect to $\left(r_{1}, r_{2}, s\right)$.

Some examples of the kinds of $(r, s)$-retracts stated in the Definition 2.6 above can be found in [30] (Example 3.1 and 3.2).

\subsection{Negations on $L$}

A natural extension of the notion of fuzzy set can be made by considering arbitrary bounded lattices as possible sets of truth values. This is the case of $L$-fuzzy sets introduced in [16] by Goguen. Lowen in 1978 proposed a notion of negation as involutive and antitonic functions [23]. In the following definition the concept of negation on a bounded lattice $L$ is presented in the sense of Trillas [37].

Definition 2.7. [33] A mapping $N: L \rightarrow L$ is a negation on $L$ or just an $L$-negation, if the following properties are satisfied for each $x, y \in L$ :

(N1) $N\left(0_{L}\right)=1_{L}$ and $N\left(1_{L}\right)=0_{L}$ and

(N2) If $x \leqslant_{L} y$ then $N(y) \leqslant_{L} N(x)$.

Moreover, the L-negation $N$ is considered strong if it also satisfies the involution property, i.e.

(N3) $N(N(x))=x$ for each $x \in L$.

The L-negation is strict if satisfies the property:

(N4) $N(x)<_{L} N(y)$ whenever $y<_{L} x$.

The L-negation $N$ is frontier if satisfies the property:

(N5) $N(x) \in\left\{0_{L}, 1_{L}\right\}$ if and only if $x=0_{L}$ or $x=1_{L}$.

Remark 3. One can easily verify that for each strong L-negation we have that $x \| y$ if and only if $N(x) \| N(y)$. Notice that although every lattice admits a negation $N$, it is not true that every lattice admits an involutive negation (see Example 2.10).

Example 2.8. Let $L$ be any lattice such that there exists $x_{0} \in L$ with $x_{0} \neq$ $0_{L}, 1_{L}$. Then the mapping:

$$
N(x)= \begin{cases}0_{L} & \text { if } x=1_{L} \\ 1_{L} & \text { if } x=0_{L} \\ x_{0} & \text { otherwise }\end{cases}
$$

is a frontier negation. Notice that this example proves that for every lattice $L$ with at least three elements, it is possible to define a frontier negation. 
Notice that an element $e \in L$ is a fixed point of an $L$-negation $N$ if $N(e)=e$. Unlike the usual case and the interval-valued case (see [3]), strong $L$-negations may not have fixed points. If it exists, the fixed point needs not be unique.

Example 2.9. Let $L$ and $M$ be bounded lattices as shown in the Figure 1 (note that $M$ is a 4 elements Boolean Algebra). The function $N_{1}: M \rightarrow M$ defined by $N_{1}\left(0_{M}\right)=1_{M}, N_{1}(x)=y, N_{1}(y)=x$ and $N_{1}\left(1_{M}\right)=0_{M}$ is a strong $M$-negation. Nevertheless, $N_{1}$ has no fixed points.

Now, consider a function $N_{2}: L \rightarrow L$ given by $N_{2}\left(0_{L}\right)=1_{L}, N_{2}(a)=e$, $N_{2}(e)=a, N_{2}\left(1_{L}\right)=0_{L}$ and $N_{2}(u)=u$ for each $u \in L \backslash\left\{a, e, 0_{L}, 1_{L}\right\}$. In this case, $N_{2}$ is a strong L-negation with three fixed points, namely b, $c$ and $d$.

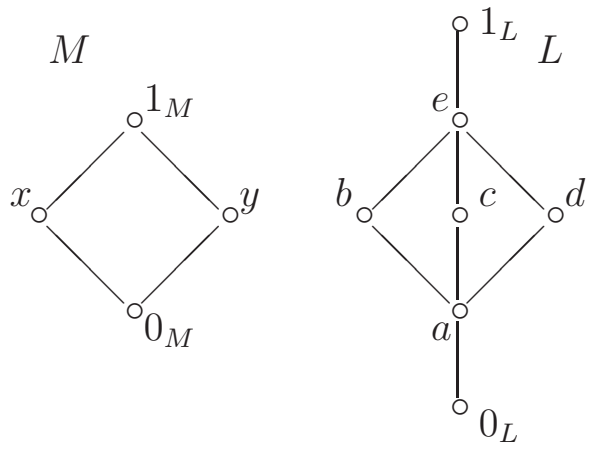

Figure 1: Hasse diagrams of lattices $M$ and $L$

Example 2.10. Consider the lattice $L_{0}$ obtained from lattice $L$ in Figure 1 by omitting the point a. Then, it does not exist a strong negation for this lattice. If $N$ is such negation, then we should consider $0_{L}<_{L} N(e)<_{L}$ $N(b), N(c), N(d)<_{L} 1_{L}$ which is not possible due to the injectivity of a strong negation.

Thus, in general we cannot define a strong negation over a lattice as shown here. This is neither due to the fact that it is considered a partial order on $L$ nor due to the fact that the lattice has a discrete structure. For instance, it is easy to see that it is not possible to define a strong negation on the linear ordered lattice $L=\{0,1\} \cup[2,3]$ as well, considering the usual linear order of real numbers $\leqslant_{L}$ on $L$.

Proposition 2.11. [4] Let $N: L \rightarrow L$ be a function, $\rho$ be an automorphism of $L$ and $i \in\{1,2,3,4,5\} . N$ satisfies (Ni) if and only if its conjugate $N^{\rho}$ 
satisfies (Ni). Moreover, $e$ is a fixed point of $N$ if and only if $\rho^{-1}(e)$ is a fixed point of $N^{\rho}$.

Corollary 2.12. Let $N: L \rightarrow L$ be a function and $\rho$ be an automorphism of L. $N$ is an (strong, strict, frontier) L-negation if and only if $N^{\rho}$ is an (strong, strict, frontier) L-negation.

\subsection{Restricted Equivalence Functions}

As it has been stated in the introduction, the concept of restricted equivalence function (REF) was given in order to obtain (by aggregation) global measures for comparing images satisfying certain conditions. Evidently, the use of these functions can be also extend to any other object which is represented by a fuzzy set. In situations where the objects are represented by an extension of fuzzy sets, such as interval-valued fuzzy sets, restricted equivalence functions must be redefined to the concrete setting. For example, in [34] the extension of mathematical morphology operators to lattice structures is studied, as well as in [26], where images are represented as interval-valued fuzzy sets. Moreover, the RGB color scheme (commonly used in color images) can be seen as a Cartesian product of lattices [5, 32]. Finally, there are other applications, such as fuzzy rule-based classification systems in which some extension of REFs have been used [36]. Since all these extensions take place in lattice theory, there are some important reasons to consider in a general way restricted equivalence functions defined on bounded lattices.

One of the most common definition of equivalence was proposed by Fodor and Roubens in [15].

Definition 2.13. A function EF : $[0,1]^{2} \rightarrow[0,1]$ is called an equivalence if it satisfies the following conditions:

1. $E F(x, y)=E F(y, x)$ for all $x, y \in[0,1]$;

2. $E F(0,1)=E F(1,0)=0$;

3. $E F(x, x)=1$ for all $x \in[0,1]$;

4. If $x \leqslant y \leqslant z \leqslant t$ then $E F(x, t) \leqslant E F(y, z)$.

Naturally, this concept can be generalized for bounded lattices as follows.

Definition 2.14. Let $L$ be a bounded lattice. A function $E F: L^{2} \rightarrow L$ is called an L-equivalence if it satisfies the following conditions: 
$(F 1) E F(x, y)=E F(y, x)$ for all $x, y \in L$;

(F2) $E F\left(0_{L}, 1_{L}\right)=E F\left(1_{L}, 0_{L}\right)=0_{L}$;

(F3) $E F(x, x)=1_{L}$ for all $x \in L$;

$(F 4)$ If $x \leqslant_{L} y \leqslant_{L} z$ then $E F(x, z) \leqslant_{L} E F(y, z)$ and $E F(x, z) \leqslant_{L} E F(x, y)$.

Nonetheless, equivalence functions as those described in Definition 2.14 do not allow us to ensure that only $\left(0_{L}, 1_{L}\right)$ and $\left(1_{L}, 0_{L}\right)$ are assigned to $0_{L}$, i.e., it could exist a pair $(x, y) \in L \backslash\left\{0_{L}, 1_{L}\right\}$ such that $E F(x, y)=0_{L}$. This is a disadvantage for comparing images since it cannot be ensured that two images in black and white are opposite if and only if one is the negative of the other. This is also a backdraw of Definition 2.13. In order to solve this problem among others, Bustince et al. in [7] redefined (on [0,1]) Fodor and Roubens equivalences by adding some constraints and introducing the concept of restricted equivalence function. A generalization for bounded lattices is given in the following definition.

Definition 2.15. Let $N$ be a strong negation on $L$. A function $R E F: L^{2} \rightarrow$ $L$ is called a restricted equivalence function on $L$ with respect to $N$, or just an $L-R E F$ with respect to $N$, if it satisfies, for all $x, y, z \in L$, the following conditions:

(L1) $R E F(x, y)=R E F(y, x)$;

(L2) $R E F(x, y)=1_{L}$ if and only if $x=y$;

(L3) $R E F(x, y)=0_{L}$ if and only if $\{x, y\}=\left\{0_{L}, 1_{L}\right\}$;

(L4) $R E F(x, y)=R E F(N(x), N(y))$;

$(L 5)$ if $x \leqslant_{L} y \leqslant_{L} z$ then $\operatorname{REF}(x, z) \leqslant_{L} \operatorname{REF}(x, y)$.

As we have discussed in Example 2.10 it is not possible to determine for which kind of lattice a strong fuzzy negation can be defined. However, for defining restricted equivalence funtions on lattice the associated fuzzy negation must be strong to ensure no conflits between properties $(L 2)$ and $(L 4)$ arise. Hence, definition 2.15 is retricted to those lattices where a strong fuzzy negation may be defined. 
Example 2.16. Let $L$ be a lattice with at least three elements, and take $x_{0} \in L \backslash\left\{0_{L}, 1_{L}\right\}$. Then we can define

$$
\operatorname{REF}(x, y)= \begin{cases}1_{L} & \text { if } x=y \\ 0_{L} & \text { if }\{x, y\}=\left\{0_{L}, 1_{L}\right\} \\ x_{0} & \text { otherwise, }\end{cases}
$$

which is a restricted equivalence function with respect to any strong negation $N$.

Notice that from $(L 4),(L 5)$ and $(L 1)$, it is also possible to conclude that $R E F(x, z) \leqslant_{L} \operatorname{REF}(y, z)$ whenever $x \leqslant_{L} y \leqslant_{L} z$.

Example 2.17. Let $M$ be the bounded lattice in Figure 1 and $N_{1}$ be a strong $M$-negation as in Example 2.9. Thus, a function REF : $M^{2} \rightarrow M$ as defined in the Table 1 is a L-REF with respect to $N_{1}$ in the sense of Definition 2.15.

\begin{tabular}{|c||c|c|c|c|}
\hline REF & $0_{L}$ & $x$ & $y$ & $1_{L}$ \\
\hline \hline $0_{L}$ & $1_{L}$ & $x$ & $y$ & $0_{L}$ \\
\hline$x$ & $x$ & $1_{L}$ & $x$ & $y$ \\
\hline$y$ & $y$ & $x$ & $1_{L}$ & $x$ \\
\hline $1_{L}$ & $0_{L}$ & $y$ & $x$ & $1_{L}$ \\
\hline
\end{tabular}

Table 1: Restricted equivalence function on lattice $M$

In this case the mapping $N(x)=R E F\left(0_{L}, x\right)$ defines a strong negation on the lattice $M$.

\section{Fuzzy Negation Constructed from REF}

The remark in Example 2.17 is just a particular case of the following general result.

Proposition 3.1. Let REF be a restricted equivalence function on the lattice $L$ with respect to some strong negation $N$. Then, the mapping

$$
N_{R E F}(x)=R E F\left(0_{L}, x\right)
$$

is a frontier negation on $L$. 
Proof: Straightforward.

Example 3.2. Notice that a given function can sometimes be an L-REF with respect to one negation but not with respect to another one. For instance, consider Example 2.17. In this case, following the notation of Proposition 3.1, we have that $N_{R E F}\left(0_{L}\right)=1_{L} ; N_{R E F}(x)=x, N_{R E F}(y)=y$ and $N_{R E F}\left(1_{L}\right)=0_{L}$, which is different from the negation $N_{1}$ considered for the definition of the REF. But for this $N_{R E F}$ we see that $R E F\left(0_{L}, x\right)=x \neq y=$ REF $\left(1_{L}, x\right)$, so property (L4) does not hold.

Corollary 3.3. Let $R E F$ be a restricted equivalence function on $L$ with respect to some strong negation $N$ and let $N_{R E F}$ be defined as in Proposition 3.1. Then, $N_{R E F}=g \circ N$ with $g: L \rightarrow L$ defined as $g(x)=R E F\left(1_{L}, x\right)$.

Example 3.4. Let's consider again the lattice L of Example 2.17. Take as $R E F$ the function defined in Example 2.16 with $x_{0}=y$. This is a restricted equivalence function with respect to any strong negation defined on $L$; therefore, in particular for $N=N_{1}$. Yet, with the notation of Proposition 3.1, we have that $N_{R E F}(x)=R E F\left(0_{L}, x\right)=y=R E F\left(0_{L}, y\right)=N_{R E F}(y)$; so $N_{R E F}$ is not injective and hence, it is not strong.

Definition 3.5. Let $R E F$ be a restricted equivalence function. $R E F$ is called strict if for each $x, y, z \in[0,1]$ such that $x<_{L} y<_{L} z, \operatorname{REF}(x, z)<_{L}$ $R E F(x, y)$.

An example of strict restricted equivalence function is the given in [7, Example 1] whereas example given in [7, Example 4] are examples of restricted equivalence functions that are not strict.

Proposition 3.6. Let $R E F$ be a strict restricted equivalence relation. Then $N_{R E F}$ is an strict negation.

Proof: Straightforward.

Lemma 3.7. If $R E F$ is a restricted equivalence function on $L$ with respect to some strong negation $N$ and $e$ is a fixed point of $N$ then $\operatorname{REF}\left(1_{L}, e\right)=$ $\operatorname{REF}\left(0_{L}, e\right)$.

Proof: $\quad R E F\left(0_{L}, e\right)=R E F\left(N\left(0_{L}\right), N(e)\right)=R E F\left(1_{L}, e\right)$. 
Proposition 3.8. Let $R E F$ be a strict restricted equivalence function on $L$ with respect to the strong negations $N_{1}$ and $N_{2}$. If $e_{1}$ and $e_{2}$ are fixed points of $N_{1}$ and $N_{2}$ respectively, such that $e_{1} \frown e_{2}$ then $e_{1}=e_{2}$.

Proof: Let $e_{1}$ and $e_{2}$ be the fixed points of $N_{1}$ and $N_{2}$, respectively. Without loss of generality we can assume that $e_{1} \leqslant{ }_{L} e_{2}$.

$$
\begin{aligned}
N_{R E F}\left(e_{1}\right) & =\operatorname{REF}\left(0_{L}, e_{1}\right) & & \text { by eq. }(3) \\
& =\operatorname{REF}\left(1_{L}, e_{1}\right) & & \text { by Lemma } 3.7 \\
& \leqslant \operatorname{REF}\left(1_{L}, e_{2}\right) & & \text { by (L5) } \\
& =\operatorname{REF}\left(N_{2}\left(1_{L}\right), N_{2}\left(e_{2}\right)\right) & & \text { by (L4) } \\
& =\operatorname{REF}\left(0_{L}, e_{2}\right) & & \\
& =N_{R E F}\left(e_{2}\right) & &
\end{aligned}
$$

So, $N_{R E F}\left(e_{1}\right) \leqslant{ }_{L} N_{R E F}\left(e_{2}\right)$. But by (L5), $N_{R E F}\left(e_{2}\right)=R E F\left(e_{2}, 0\right) \leqslant{ }_{L}$ $R E F\left(e_{1}, 0\right)=N_{R E F}\left(e_{1}\right)$ and hence, $N_{R E F}\left(e_{1}\right)=N_{R E F}\left(e_{2}\right)$. Therefore, by Proposition $3.6 N_{R E F}$ is strict and hence $e_{1}=e_{2}$.

\section{Extension of Restricted Equivalence Functions}

This section starts with the application of two different methods for extending fuzzy operators defined in previous works [27, 30]. In [27] it was introduced an extension method via retractions that generalizes the extension proposed by Saminger-Platz et al. in [35]. In [30] we proposed a new extension method, called extension method via e-operators, which was aimed at achieving more efficiency to preserve the properties of extended operators.

\subsection{Extension via Retractions}

Let $M$ be a complete ordinary sublattice of $L$ and $T^{M}$ be a t-norm on $M$. Under these conditions, Saminger-Platz et al. in [35] have proposed a method for extending a t-norm $T$ from $M$ to $L$ in a natural but drastic and particular way since it collapses all elements of $L \backslash M$ on $M$ and only considers complete sublattices.

Seeking to propose a more general and flexible extension method, Palmeira and Bedregal presented in $[27,28,31]$ another way to extend t-norms, tconorms and fuzzy negations considering $(r, s)$-sublattices which generalizes the extension proposed in [35]. 
Proposition 4.1. [27] Let $M<L$ with respect to $(r, s)$. If $T$ is a $t$-norm on $M$ then $T^{E}: L \times L \longrightarrow L$ defined by

$$
T^{E}(x, y)=\left\{\begin{aligned}
x \wedge_{L} y, & \text { if } 1_{L} \in\{x, y\} \\
s(T(r(x), r(y))), & \text { otherwise }
\end{aligned}\right.
$$

is a t-norm which extends $T$ from $M$ to $L$.

In a similar way, it is possible to extend t-conorms (see Proposition 4.1 in [27]). For negations we have

Proposition 4.2. [27] Let $M$ be a $(r, s)$-retract of $L$ and $N: M \longrightarrow M$ be a fuzzy negation. Then $N^{E}(x)=s(N(r(x)))$ for each $x \in L$ is a fuzzy negation that extends $N$ from $M$ to $L$.

It is worth noting that, in Proposition 4.1, $r$ must be a lower retraction, whereas in Proposition 4.2, it is arbitrary (i.e., it could be lower, upper or neither). This fact shows negations can be extended in a more flexible way.

An advantage of this extension method is that it can be applied for several operators in the framework of fuzzy logic and applications. We have investigated the behavior of this method for t-subnorms, lattice automorphisms and some other particular classes of fuzzy operators (see $[28,31]$ ).

Theorem 4.3. Let $M$ be $a(r, s)$-retract of $L$ and $E F: M^{2} \rightarrow M$ an equivalence function. Then the function

$$
E F^{E}(x, y)=s(E F(r(x), r(y)))
$$

for each pair $(x, y) \in L^{2}$ is an equivalence function that extends $E F$ from $M$ to $L$.

Proof: It is clear that $E F^{E}$ satisfies $(F 1)$ since $E F$ is an equivalence function. Moreover, for all $x \in L$ we have

$$
E F^{E}(x, x)=s(E F(r(x), r(x)))=s\left(1_{M}\right)=1_{L}
$$

and

$$
E F^{E}\left(0_{L}, 1_{L}\right)=s\left(E F\left(r\left(0_{L}\right), r\left(1_{L}\right)\right)\right)=s\left(E F\left(0_{M}, 1_{L}\right)\right)=s\left(0_{M}\right)=0_{L}
$$


Analogously, one can prove that $E F^{E}\left(1_{L}, 0_{L}\right)=0_{L}$. Thus, $(F 2)$ and $(F 3)$ hold.

It remains to prove $(F 4)$. To do so, note that for all $x, y, z \in L$ such that $x \leqslant_{L} y \leqslant_{L} z$ it follows that $r(x) \leqslant_{M} r(y) \leqslant_{M} r(z)$ and hence $E F(r(x), r(z)) \leqslant_{M}$ $E F(r(x), r(y))$. Thus

$$
E F^{E}(x, z)=s(E F(r(x), r(z))) \leqslant{ }_{L} s(E F(r(x), r(y)))=E F^{E}(x, y) .
$$

Notice that Definition 2.15 refines Definition 2.14, since it imposes some new constraints. Aside from property $(L 4)$, Definition 2.15 also requires that the unique elements assigned to $0_{L}$ by $R E F$ are $\left(0_{L}, 1_{L}\right)$ and $\left(1_{L}, 0_{L}\right)$ and that $R E F$ is evaluated as $1_{L}$ only for pairs with the same value in both coordinates. Thus, it reveals that we cannot extend restricted equivalence functions using the method provided by Propositions 4.1 and 4.2.

If $M$ is a $(r, s)$-retract of $L, N$ is a strong fuzzy negation on $M$ and $R E F: M^{2} \rightarrow M$ a restricted equivalence function with respect to $N$ then the function $R E F^{E}: L^{2} \rightarrow L$ given by $R E F^{E}(x, y)=s(R E F(r(x), r(y)))$ satisfies property $(L 4)$

$$
\begin{aligned}
R E F^{E}\left(N^{E}(x), N^{E}(y)\right) & =s\left(\operatorname{REF}\left(r\left(N^{E}(x)\right), r\left(N^{E}(y)\right)\right)\right) \\
& =s(\operatorname{REF}(r(s(N(r(x)))), r(s(N(r(y)))))) \\
& =s(\operatorname{REF}(N(r(x)), N(r(y)))) \\
& =s(\operatorname{REF}(r(x), r(y))) \\
& =\operatorname{REF}^{E}(x, y)
\end{aligned}
$$

Moreover, if $r$ is such that $r(x)=0_{L}$ if and only if $x=0_{L}$ and $r(x)=$ $1_{L}$ if and only if $x=1_{L}$ then supposing $\operatorname{REF}^{E}(x, y)=0_{L}$ we have that $s(R E F(r(x), r(y)))=0_{L}$. Since $s$ is an injective function it follows that $\operatorname{REF}(r(x), r(y))=0_{M}$ and hence $r(x)=1_{M}$ and $r(y)=0_{M}$ or $r(x)=0_{M}$ and $r(y)=1_{M}$ by $(L 3)$, what allows us to conclude that $x=1_{L}$ and $y=0_{L}$ or $x=0_{L}$ and $y=1_{L}$, i.e. $R E F^{E}$ satisfies property ( $\left.L 3\right)$ (the necessity side of (L3) is shown in Theorem 4.3). Nevertheless $R E F^{E}$ does not satisfy (L2), in general, as the following example shows.

Example 4.4. Let $M$ and $L$ be the bounded lattices depicted in Figure 1. Then function $s: M \rightarrow L$ defined by $s\left(0_{M}\right)=0_{L}, s(x)=b, s(y)=d$ and 
$s\left(1_{M}\right)=1_{L}$ is a pseudo-inverse of the retraction

$$
r(t)=\left\{\begin{array}{cl}
0_{M}, & t=0_{L} \\
1_{M}, & t=1_{L} \\
y, & t=d \\
x, & \text { otherwise. }
\end{array}\right.
$$

and hence $M$ is a $(r, s)$-retract of $L$. Then, considering the REF as in Example 2.17, its extension to $L$ is such that $\operatorname{REF}^{E}(e, c)=s(R E F(r(e), r(c)))=$ $s(R E F(x, x))=s\left(1_{M}\right)=1_{L}$ showing that $R E F^{E}$ does not satisfies (L2).

The above discussion allows us to enunciate the following proposition (extension of a weak version of restricted equivalence functions).

Proposition 4.5. Let $M$ be an $(r, s)$-retract of $L, N$ be a fuzzy negation on $M$ and REF : $M^{2} \rightarrow M$ a restricted equivalence function with respect $N$. Then, $R E F^{E}: L^{2} \rightarrow L$ given by

$$
R E F^{E}(x, y)=s(R E F(r(x), r(y)))
$$

satisfies (L1), (L4) with respect $N^{E},(L 5)$ and $(W L 2) R E F^{E}(x, x)=1_{L}$ for all $x, y \in L$. Moreover, if

$$
r(x)=0_{M} \text { if and only if } x=0_{L}
$$

and

$$
r(x)=1_{M} \text { if and only if } x=1_{L}
$$

then (L3) holds.

Notice that property (L2) holds for the L-REF defined in Equation (6) only when $M$ and $L$ are isomorphic once otherwise there exist $x, y \in L$ such that $x \neq y$ and $r(x)=r(y)$ imply $R E F^{E}(x, y)=s(R E F(r(x), r(y)))=$ $s\left(1_{M}\right)=1_{L}$. This is the motivation for defining the following weakened notion of restricted equivalence functions.

Definition 4.6. Let $L$ be an arbitrary bounded lattice. A function REF : $L^{2} \rightarrow L$ satisfying properties (L1), (WL2), (L3), (L4) and (L5) is called a weak restricted equivalence function. 
Proposition 4.7. Let $M$ be a $(r, s)$-retract of $L, N$ be a fuzzy negation on $M$ and REF : $M^{2} \rightarrow M$ a weak restricted equivalence function with respect $N$. If $r$ satisfies Equations (7) and (8) then $R E F^{E}: L^{2} \rightarrow L$ given as in Equation (6) is a weak restricted equivalence function with respect $N^{E}$.

The idea behind Proposition 4.8 below is that, although it is not possible to extend REFs using the extension method via retractions, negations generated from the (weak) restricted equivalence functions can be extended.

Proposition 4.8. Let $M$ be a $(r, s)$-retract of $L$. If $R E F$ is a weak restricted equivalence function on $M$ with respect to $N$ then $N_{R E F E}(x)=$ $s\left(R E F\left(0_{M}, r(x)\right)\right)$ for each $x \in L$ is a fuzzy negation which satisfies $\left(N_{R E F}\right)^{E}=$ $N_{R E F^{E}}$.

Proof: Straghtforward.

\subsection{Extension via e-operators}

As we have seen in the previous subsection, the extension method proposed in [27] is not good enough to extend restricted equivalence functions. Similar problems arise when we try to verify which properties of fuzzy connectives are preserved by extension via retractions. For instance, Archimedian t-norms, as well as strong fuzzy negations are not preserved.

Seeking to develop a extension method more efficient in preserving the properties we have provided in [30] a method based in the idea of interval constructor using e-operator.

Definition 4.9. Let be $M \unlhd L$ with respect to $\left(r_{1}, r_{2}, s\right)$. A mapping $\odot$ : $M \times M \longrightarrow L$ is called an e-operator on $M$ if it is isotonic and satisfies the following conditions for each $a, b \in M$ and for each $x \in L$ :

$$
\begin{gathered}
r_{1}(a \odot b)=a \wedge_{M} b \text { and } r_{2}(a \odot b)=a \vee_{M} b \\
r_{1}(x) \odot r_{2}(x)=x
\end{gathered}
$$

In other words, if $M \unlhd L$ with respect to $\left(r_{1}, r_{2}, s\right)$ (by Definition 2.6, there are two retractions $r_{1}, r_{2}: L \longrightarrow M$ with the same pseudo-inverse $s: M \longrightarrow L$ such that $\left.s \circ r_{1} \leqslant i d_{L} \leqslant s \circ r_{2}\right)$ the $e$-operator $\odot$ describes an isotonic way to relate retractions $r_{1}$ and $r_{2}$ with the meet and join operators of $M$, respectively, by (9). 
Example 4.10. Let us see how to consider e-operators for extending $[0,1]$ into the interval-valued setting. Let $M=[0,1]$ with the usual order relation of numbers and let $L$ be the set of all closed subintervals of the interval $[0,1]$ $\left(L=\{\mathbf{x}=[\underline{x}, \bar{x}] \mid 0 \leq \underline{x} \leq \bar{x} \leq 1\}\right.$ with the order relation $\leq_{L}$ given by: for each $\mathbf{x}, \mathbf{y} \in L$

$$
\mathbf{x} \leq_{L} \mathbf{y} \text { if and only if } \underline{x} \leq \underline{y} \text { and } \bar{x} \leq \bar{y} .
$$

Then, we may define retractions $r_{1}, r_{2}: L \rightarrow M$ by $r_{1}(\mathbf{x})=\underline{x}$ and $r_{2}(\mathbf{x})=\bar{x}$ and $s: M \rightarrow L$ by $s(x)=[x, x]$. Therefore, $M \unlhd L$ with respect to $\left(r_{1}, r_{2}, s\right)$ and an e-operator can be defined by $x \odot y=[x \wedge y, x \vee y]$.

Lemma 4.11. [30] Consider $M \unlhd L$ with respect to $\left(r_{1}, r_{2}, s\right)$ and let $\odot$ be an e-operator on $M$. Then, for all $a, b \in M$ and $x, y \in L$, the following properties hold:

1. $a \leqslant_{M} b$ if and only if $r_{1}(a \odot b)=a$ and $r_{2}(a \odot b)=b$;

2. For every $a \in M$ we have $s(a)=a \odot a$;

3.

$$
r_{1}(x) \leqslant_{M} r_{1}(y) \text { and } r_{2}(x) \leqslant_{M} r_{2}(y) \text { iff } x \leqslant_{L} y ;
$$

4. $r_{1}(x)=r_{1}(y)$ and $r_{2}(x)=r_{2}(y)$ if and only if $x=y$;

5. $\odot$ is commutative.

Proposition 4.12. [30] Consider $M \unlhd L$ with respect to $\left(r_{1}, r_{2}, s\right)$ and a mapping $\odot: M \times M \longrightarrow L$ satisfying equation (9). Then $\odot$ is an e-operator if and only if Equation (11) is satisfied.

Now, we have conditions to define the extension of t-norms and fuzzy negations via e-operator.

Theorem 4.13. [30] Let $M \unlhd L$ with respect to $\left(r_{1}, r_{2}, s\right)$ and $\odot$ be an $e$ operator on $M$. Given a $t$-norm $T$ on $M$, the function $T_{\odot}^{E}: L^{2} \longrightarrow L$ defined by

$$
T_{\odot}^{E}(x, y)=T\left(r_{1}(x), r_{1}(y)\right) \odot T\left(r_{2}(x), r_{2}(y)\right)
$$

is a t-norm on $L$.

Proposition 4.14. Let $M \unlhd L$ with respect to $\left(r_{1}, r_{2}, s\right)$ and $\odot$ an e-operator on $M$. If $N$ is a fuzzy negation on $M$ then

$$
N_{\odot}^{E}(x)=N\left(r_{1}(x)\right) \odot N\left(r_{2}(x)\right)
$$

is a fuzzy negation on L. Moreover, 
1. If $N$ is strong, then $N_{\odot}^{E}$ is also strong;

2. If $N$ is strict then $N_{\odot}^{E}$ is also strict;

3. If a is a fixed point of fuzzy negation $N$, then $s(a)$ is a fixed point of $N_{\odot}^{E}$.

Proof: Notice that items (1) and (3) are already proved in [30]. Thus, it remains to prove only item (2). To do so, suppose that $N$ is a strict negation and $x<_{L} y$ then by the item 3 of Lemma 4.11, $r_{1}(x) \leqslant_{M} r_{1}(y)$ $r_{2}(x) \leqslant_{M} r_{2}(y)$. Besides, taking into account item 4 of Lemma 4.11 and the fact that $x<_{L} y$ then both cannot be equal and $r_{1}(x)<_{M} r_{1}(y)$ or $r_{2}(x)<_{M} r_{2}(y)$. Suppose $r_{1}(x)<_{M} r_{1}(y)$, then

$$
\begin{array}{rl}
r_{1}\left(N_{\odot}^{E}(y)\right) & =r_{1}\left(N\left(r_{1}(y)\right) \odot N\left(r_{2}(y)\right)\right) \\
= & N\left(r_{2}(y)\right) \\
\leqslant_{M} & N\left(r_{2}(x)\right)=r_{1}\left(N_{\odot}^{E}(x)\right)
\end{array}
$$

and

$$
\begin{aligned}
r_{2}\left(N_{\odot}^{E}(y)\right) & =r_{2}\left(N\left(r_{1}(y)\right) \odot N\left(r_{2}(y)\right)\right) \\
& =N\left(r_{1}(y)\right) \\
& <_{M} \quad N\left(r_{1}(x)\right)=r_{2}\left(N_{\odot}^{E}(x)\right)
\end{aligned}
$$

Again by item 4. of Lemma 4.11 we have $N_{\odot}^{E}(x) \neq N_{\odot}^{E}(y)$. Consequently, since $N_{\odot}^{E}(y) \leqslant_{L} N_{\odot}^{E}(x)$ due to $x<_{L} y$ it holds $N_{\odot}^{E}(y)<_{L} N_{\odot}^{E}(x)$ i.e. $N_{\odot}^{E}$ is strict.

The case for $r_{2}(x)<_{M} r_{2}(y)$ is analogous.

Lemma 4.15. Let be $M \unlhd L$ with respect to $\left(r_{1}, r_{2}, s\right)$ and let $\odot: M \times M \rightarrow L$ be an e-operator. For each $a, b \in M$,

1. If $a \odot b=0_{L}$ then $a=0_{M}$ and $b=0_{M}$;

2. If $a \odot b=1_{L}$ then $a=1_{M}$ and $b=1_{M}$.

\section{Proof:}

1. Supposing $a \odot b=0_{L}$ we have that $a \wedge_{M} b=r_{1}(a \odot b)=r_{1}\left(0_{L}\right)=0_{M}$ and $a \vee_{M} b=r_{2}(a \odot b)=r_{1}\left(0_{L}\right)=0_{M}$, what means that $a=0_{M}$ and $b=0_{M}$.

2. Analogous to item 1. 
Theorem 4.16. Let be $M \unlhd L$ with respect to $\left(r_{1}, r_{2}, s\right)$ and let $\odot: M \times M \rightarrow$ $L$ be an e-operator. If REF : $M^{2} \rightarrow M$ is a restricted equivalence function with respect to a strong negation $N$, then the function $R E F_{\odot}^{E}: L^{2} \rightarrow L$ given by

$$
R E F_{\odot}^{E}(x, y)=R E F\left(r_{1}(x), r_{1}(y)\right) \odot R E F\left(r_{2}(x), r_{2}(y)\right)
$$

for all $x, y \in L$, is a restricted equivalence function on $L$ with respect to $N_{\odot}^{E}$.

Proof: It is easy to see that $R E F_{\odot}^{E}$ is commutative since $\odot$ is a commutative operator. Hence $(L 1)$ holds.

Note that by Lemma 4.11 (item 2) and (L2) we have that $R E F_{\odot}^{E}(x, x)=$ $R E F\left(r_{1}(x), r_{1}(x)\right) \odot R E F\left(r_{2}(x), r_{2}(x)\right)=1_{M} \odot 1_{M}=s\left(1_{M}\right)=1_{L}$. On the other hand, if $R E F_{\odot}^{E}(x, y)=1_{L}$ it follows that $R E F\left(r_{1}(x), r_{1}(y)\right) \odot$ $\operatorname{REF}\left(r_{2}(x), r_{2}(y)\right)=1_{L}$ and then by Lemma 4.15 we have that $R E F\left(r_{1}(x), r_{1}(y)\right)=$ $1_{M}$ and $R E F\left(r_{2}(x), r_{2}(y)\right)=1_{M}$. Since $R E F$ is a restricted equivalence function then $r_{1}(x)=r_{1}(y)$ and $r_{2}(x)=r_{2}(y)$. Therefore, by Lemma 4.11 we can conclude that $x=y$.

(L3)

It is clear that

$$
\begin{aligned}
R E F_{\odot}^{E}\left(1_{L}, 0_{L}\right) & =\operatorname{REF}\left(r_{1}\left(1_{L}\right), r_{1}\left(0_{L}\right)\right) \odot \operatorname{REF}\left(r_{2}\left(1_{L}\right), r_{2}\left(0_{L}\right)\right) \\
& =\operatorname{REF}\left(1_{M}, 0_{M}\right) \odot \operatorname{REF}\left(1_{M}, 0_{L}\right)=0_{M} \odot 0_{M} \\
& =s\left(0_{M}\right)=0_{L}
\end{aligned}
$$

Similarly it holds that $R E F_{\odot}^{E}\left(0_{L}, 1_{L}\right)=0_{L}$.

Conversely assume that $R E F_{\odot}^{E}(x, y)=0_{L}$. We have that $R E F\left(r_{1}(x), r_{1}(y)\right) \odot$ $\operatorname{REF}\left(r_{2}(x), r_{2}(y)\right)=0_{L}$ and then $\operatorname{REF}\left(r_{1}(x), r_{1}(y)\right)=0_{M}$ and $\operatorname{REF}\left(r_{2}(x), r_{2}(y)\right)=$ $0_{M}$ by Lemma 4.15 . Hence, by Lemma 4.11 we have

(i) $r_{1}(x)=1_{M}$ and $r_{1}(y)=0_{M}$; or

(ii) $r_{1}(x)=0_{M}$ and $r_{1}(y)=1_{M}$; and

(iii) $r_{2}(x)=1_{M}$ and $r_{2}(y)=0_{M}$; or

(iv) $r_{2}(x)=0_{M}$ and $r_{2}(y)=1_{M}$.

Note that (i) and (iv) cannot happen simultaneously, since otherwise there should be $0_{M}=r_{2}(x)<_{M} r_{1}(x)=1_{M}$ what is a contradiction with the fact that $r_{1}(x) \leqslant{ }_{M} r_{2}(x)$ for every $x \in L$. A similar argument works to show that 
(ii) and (iii) cannot happen simultaneously. It remains to analyze two other possibilities:

(i) and (iii)

In this case, by Lemma 4.11, item 4, we have that

$$
\left\{\begin{array}{l}
r_{1}(x)=1_{M}=r_{1}\left(1_{L}\right) \\
r_{2}(x)=1_{M}=r_{2}\left(1_{L}\right)
\end{array} \Rightarrow x=1_{L}\right.
$$

and

$$
\left\{\begin{array}{l}
r_{1}(y)=0_{M}=r_{1}\left(0_{L}\right) \\
r_{2}(y)=0_{M}=r_{2}\left(0_{L}\right)
\end{array} \Rightarrow y=0_{L}\right.
$$

Analogously, it can be proved that (ii) and (iv) imply $x=0_{L}$ and $y=1_{L}$. $(L 4)$

Let $N$ be a strict negation on $M$ and hence $N_{\odot}^{E}$ is a strict negation on L by item 2. of Proposition 4.14. We shall prove that $R E F_{\odot}^{E}(x, y)=R E F_{\odot}^{E}\left(N_{\odot}^{E}(x), N_{\odot}^{E}(y)\right)$ for each $x, y \in L$. Indeed, we have that

$$
\begin{aligned}
R E F_{\odot}^{E}\left(N_{\odot}^{E}(x), N_{\odot}^{E}(y)\right)= & \operatorname{REF}\left(r_{1}\left(N^{E}(x)\right), r_{1}\left(N^{E}(y)\right)\right) \odot \\
& \operatorname{REF}\left(r_{2}\left(N^{E}(x)\right), r_{2}\left(N^{E}(y)\right)\right)
\end{aligned}
$$

Since by Lemma 4.11 item 1

$$
\begin{aligned}
\operatorname{REF}\left(r_{1}\left(N^{E}(x)\right), r_{1}\left(N^{E}(y)\right)\right)= & \operatorname{REF}\left(r_{1}\left(N\left(r_{1}(x)\right) \odot N\left(r_{2}(x)\right)\right),\right. \\
& \left.r_{1}\left(N\left(r_{1}(y)\right) \odot N\left(r_{2}(y)\right)\right)\right) \\
= & R E F\left(N\left(r_{2}(x)\right), N\left(r_{2}(y)\right)\right)
\end{aligned}
$$

and

$$
\begin{aligned}
\operatorname{REF}\left(r_{2}\left(N^{E}(x)\right), r_{2}\left(N^{E}(y)\right)\right)= & \operatorname{REF}\left(r_{2}\left(N\left(r_{1}(x)\right) \odot N\left(r_{2}(x)\right)\right),\right. \\
& \left.r_{2}\left(N\left(r_{1}(y)\right) \odot N\left(r_{2}(y)\right)\right)\right) \\
= & R E F\left(N\left(r_{1}(x)\right), N\left(r_{1}(y)\right)\right)
\end{aligned}
$$

Based on the commutativity of $\odot$ and Equation (15), it follows that

$$
\begin{aligned}
& R E F_{\odot}^{E}\left(N_{\odot}^{E}(x), N_{\odot}^{E}(y)\right)=R E F\left(N\left(r_{1}(x)\right), N\left(r_{1}(y)\right)\right) \odot R E F\left(N\left(r_{2}(x)\right), N\left(r_{2}(y)\right)\right) \\
& =R E F\left(r_{1}(x), r_{1}(y)\right) \odot R E F\left(r_{2}(x), r_{2}(y)\right) \\
& =R E F_{\odot}^{E}(x, y)
\end{aligned}
$$

Let $x, y, z \in L$ such that $x \leqslant_{L} y \leqslant_{L} z$. In this case we have $r_{1}(x) \leqslant_{M}$ $r_{1}(y) \leqslant_{M} r_{1}(z)$ and $r_{2}(x) \leqslant_{M} r_{2}(y) \leqslant_{M} r_{2}(z)$ and hence $R E F\left(r_{1}(x), r_{1}(z)\right) \leqslant_{M}$ 
$\operatorname{REF}\left(r_{1}(x), r_{1}(y)\right)$ and $\operatorname{REF}\left(r_{2}(x), r_{2}(z)\right) \leqslant_{M} \operatorname{REF}\left(r_{2}(x), r_{2}(y)\right)$. Then, from the isotonicity of $\odot$ we have that

$$
\begin{aligned}
R E F_{\odot}^{E}(x, z) & =\operatorname{REF}\left(r_{1}(x), r_{1}(z)\right) \odot \operatorname{REF}\left(r_{2}(x), r_{2}(z)\right) \\
& \leqslant_{L} \operatorname{REF}\left(r_{1}(x), r_{1}(y)\right) \odot \operatorname{REF}\left(r_{2}(x), r_{2}(y)\right) \\
& =\operatorname{REF} \odot(x, y)
\end{aligned}
$$

Example 4.17. Let REF : $[0,1]^{2} \rightarrow[0,1]$ be the restricted equivalence function given by

$$
\operatorname{REF}(x, y)=1-|x-y|
$$

with respect to the standard negation, introduced in [7]. So, taking the eoperator $\odot: M \times M \rightarrow L$ introduced in 4.10, we have the following restricted equivalence function on $L$

$$
\begin{aligned}
R E F_{\odot}^{E}(\mathbf{x}, \mathbf{y})= & \operatorname{REF}\left(r_{1}(\mathbf{x}), r_{1}(\mathbf{y})\right) \odot \operatorname{REF}\left(r_{2}(\mathbf{x}), r_{2}(\mathbf{y})\right) \\
= & {[(1-|\underline{x}-\underline{y}|) \wedge(1-|\bar{x}-\bar{y}|),} \\
& (1-|\underline{x}-\underline{y}|) \vee(1-|\bar{x}-\bar{y}|)]
\end{aligned}
$$

\subsection{Extension of negations obtained from a REF}

Now we turn our attention to a special class of fuzzy negations constructed from restricted equivalence functions, as defined in Section 3.

Notice that if $M$ is a $\left(r_{1}, r_{2}, s\right)$-retract of $L$, given a restricted equivalence function $R E F$ on $M$, its extension using e-operators is naturally an extension for the negation obtained from this $R E F$, i.e. an extension of $N_{R E F}(x)=$ $R E F\left(0_{M}, x\right)$ which is given by

$$
\begin{aligned}
\left(N_{R E F}\right)_{\odot}^{E}(y) & =N_{R E F}\left(r_{1}(y)\right) \odot N_{R E F}\left(r_{2}(y)\right) \\
& =\operatorname{REF}\left(0_{M}, r_{1}(y)\right) \odot \operatorname{REF}\left(0_{M}, r_{2}(y)\right)
\end{aligned}
$$

for all $y \in L$. This is obviously a fuzzy negation on $L$ (see Section 4 ).

On the other hand, we have that

$$
\begin{aligned}
N_{R E F_{\odot}^{E}}(y) & =\operatorname{REF} F_{\odot}^{E}\left(0_{L}, y\right) \\
& =\operatorname{REF}\left(r_{1}\left(0_{L}\right), r_{1}(y)\right) \odot \operatorname{REF}\left(r_{2}\left(0_{L}\right), r_{2}(y)\right) \\
& =\operatorname{REF}\left(0_{M}, r_{1}(y)\right) \odot \operatorname{REF}\left(0_{M}, r_{2}(y)\right)
\end{aligned}
$$

So, it follows that $\left(N_{R E F}\right)_{\odot}^{E}=N_{R E F_{\odot}^{E}}$. 


\section{Restricted Dissimilarity Function}

According to the literature, there are different ways to define functions to measure similarity or dissimilarity between two images. Bustince et al. [10] provided the notion of restricted dissimilarity functions based on error metric for images proposed by [1].

Definition 5.1. Let $L$ be a bounded lattice. A function $d_{L}: L^{2} \rightarrow L$ is called a restricted dissimilarity function on $L$ (for short $L-R D F$ ) if it satisfies, for all $x, y, z \in L$, the following conditions:

$(D 1) d_{L}(x, y)=d_{L}(y, x)$;

(D2) $d_{L}(x, y)=1_{L}$ if and only if either $x=1_{L}$ and $y=0_{L}$ or $x=0_{L}$ and $y=1_{L}$;

(D3) $d_{L}(x, y)=0_{L}$ if and only if $x=y$;

$(D 4)$ if $x \leqslant_{L} y \leqslant_{L} z$ then $d_{L}(x, y) \leqslant_{L} d_{L}(x, z)$ and $d_{L}(y, z) \leqslant_{L} d_{L}(x, z)$.

As we have made for restricted equivalence functions, here we apply both extension methods via retractions and via e-operators to extend restricted dissimilarity functions.

Proposition 5.2. Let $M$ be a $(r, s)$-retract of $L$ and suppose $r$ is such that $r(x)=0_{M}$ if and only if $x=0_{L}$ and $r(x)=1_{M}$ if and only if $x=$ $1_{L}$. If $d_{M}: M^{2} \rightarrow M$ a restricted dissimilarity function, then $d_{M}^{E}(x, y)=$ $s\left(d_{M}(r(x), r(y))\right)$ for each $x, y \in L$ satisfies $(D 1)$, (D2) and (D4). Moreover, $d_{L}(x, x)=0_{L}$ for every $x \in L$.

Proof: It is clear that $(D 1)$ holds from commutativity of $d_{M}$. Moreover, for every $x \in L$ it follows that $d_{M}^{E}(x, x)=s\left(d_{M}(r(x), r(x))\right)=s\left(0_{M}\right)=0_{L}$. So, it remains to prove $(D 2)$ and $(D 4)$.

Supposing $d_{M}^{E}(x, y)=1_{L}$ then we have that $s\left(d_{M}(r(x), r(y))\right)=1_{L}$ which implies $d_{M}(r(x), r(y))=r\left(1_{L}\right)=1_{M}$ and hence either $r(x)=1_{M}$ and $r(y)=$ $0_{M}$ or $r(x)=0_{M}$ and $r(y)=1_{M}$. Since $r(x)=0_{M}$ if and only if $x=0_{L}$ and $r(x)=1_{M}$ if and only if $x=1_{L}$ then either $x=1_{L}$ and $y=0_{L}$ or $x=0_{L}$ and $y=1_{L}$. On the other hand, $d_{M}^{E}\left(1_{L}, 0_{L}\right)=s\left(d_{M}\left(r\left(1_{L}\right), r\left(0_{M}\right)\right)\right)=$ $s\left(d_{M}\left(1_{M}, 0_{M}\right)\right)=s\left(1_{M}\right)=1_{L}$. By item 1 it holds that $d_{M}^{E}\left(0_{L}, 1_{L}\right)=0_{L}$. Therefore, $d_{M}^{E}$ satisfies $(D 2)$.

Now, take $x, y, z \in L$ such that $x \leqslant_{L} y \leqslant_{L} z$. In this case, we have that $r(x) \leqslant_{M} r(y) \leqslant_{M} r(z)$ and hence $d_{M}(x, y) \leqslant_{L} d_{M}(x, z)$. Then

$$
d_{M}^{E}(x, y)=s\left(d_{M}(r(x), r(y))\right) \leqslant_{L} s\left(d_{M}(r(x), r(z))\right)=d_{M}^{E}(x, z)
$$


Analogously, one can prove that $d_{M}^{E}(y, z) \leqslant_{L} d_{M}^{E}(x, z)$, so $(D 4)$ holds.

In other words, Proposition 5.2 says that the extension method via retractions is not efficient to extend restricted dissimilarity functions, since only a weak version of this kind of function is recovered. The same happened for the extension of restricted equivalence functions with this method, as seen in Section 4.1. Using the extension method via e-operators, though, makes possible to extend restricted dissimilarity functions successfully.

Proposition 5.3. Let $M \unlhd L$ with respect to $\left(r_{1}, r_{2}, s\right), \odot: M \times M \rightarrow L$ be an e-operator and $d_{M}: M^{2} \rightarrow M$ a restricted dissimilarity function. Then the function $\left(d_{M}\right)_{\odot}^{E}: L^{2} \rightarrow L$ given by

$$
\left(d_{M}\right)_{\odot}^{E}(x, y)=d_{M}\left(r_{1}(x), r_{1}(y)\right) \odot d_{M}\left(r_{2}(x), r_{2}(y)\right)
$$

for all $x, y \in L$, is a restricted dissimilarity function on $L$.

Proof: Similar to proof of Theorem 4.14.

The following theorem proposes a way to construct restricted dissimilarity functions from restricted equivalence functions.

Theorem 5.4. Let REF : $L^{2} \rightarrow L$ be an $L-R E F$ with respect to $N$. If $N^{\prime}$ a frontier L-negation (not necessarily equal to $N$ ) then, the function defined by

$$
d_{L}(x, y)=N^{\prime}(R E F(x, y)) \text { for all } x, y \in L
$$

is a restricted dissimilarity function.

Proof: Analogously to the proof of Theorem 5 in [8].

Corollary 5.5. Let $M \unlhd L$ with respect to $\left(r_{1}, r_{2}, s\right), \odot: M \times M \rightarrow L$ be an e-operator and REF : $M^{2} \rightarrow M$ a restricted equivalence function with respect to a negation $N$. If $N^{\prime}$ is a frontier fuzzy negation on $M$, then $d_{L}: L^{2} \rightarrow L$ given by $d_{L}(x, y)=N_{\odot}^{\prime E}\left(R E F_{\odot}^{E}(x, y)\right)$ for all $x, y \in L$, is a restricted dissimilarity function.

Proof: Straightforward from Proposition 4.14, Theorem 4.16 and Theorem 5.4 . 


\section{Normal $E_{e, N}$-functions}

In this section we turn our attention to study the extension of the Normal $E_{e, N}$-funtions and its properties preserved by the extension method. Normal $E_{e, N}$-funtions are general comparison operators that consider the fixed point of a given negation.

It is known that not every strong fuzzy negation $N$ defined on a bounded lattice has a fixed point $e$. But if such point exists, the concept of normal $E_{e, N}$-functions can be defined as follows.

Definition 6.1. [8] Let $e$ be a fixed point of a strong L-negation N. A function $E_{e, N}: L \rightarrow L$ is called a normal $E_{e, N}$-function associated to $N$ if it satisfies the following conditions:

1. $E_{e, N}(x)=1_{L}$ if and only if $x=e$;

2. $E_{e, N}(x)=0_{L}$ if and only if $x=0_{L}$ or $x=1_{L}$;

3. For all $x, y \in L$ such that either $e \leqslant_{L} x \leqslant_{L} y$ or $y \leqslant_{L} x \leqslant_{L}$ e it follows $E_{e, N}(y) \leqslant L E_{e, N}(x)$;

4. $E_{e, N}(x)=E_{e, N}(N(x))$ for all $x \in L$.

Proposition 6.2. Let $M$ be a $(r, s)$-retract of $L$ and $e$ be a fixed point of a strong negation $N: M \rightarrow M$. If $E_{e, N}: M \rightarrow M$ is a normal $E_{e, N}$-function associated to $N$ then the function given by $\left(E_{e, N}\right)^{E}(x)=s\left(E_{e, N}(r(x))\right)$ for each $x \in L$ satisfies

1. $\left(E_{e, N}\right)^{E}(s(e))=1_{L}$;

2. if $r$ is a lower retraction and $\left(E_{e, N}\right)^{E}(x)=1_{L}$ then $s(e) \leqslant_{L} x$;

3. if $r$ is an upper retraction and $\left(E_{e, N}\right)^{E}(x)=1_{L}$ then $x \leqslant_{L} s(e)$;

4. if $r(x)=0_{M}$ if and only if $x=0_{L}$ and $r(x)=1_{M}$ if and only if $x=1_{L}$ then $\left(E_{e, N}\right)^{E}(x)=0_{L}$ if and only if $x=0_{L}$ or $x=1_{L}$;

5. For all $x, y \in L$ such that either $s(e) \leqslant_{L} x \leqslant_{L} y$ or $y \leqslant_{L} x \leqslant_{L} s(e)$ it follows $\left(E_{e, N}\right)^{E}(y) \leqslant_{L}\left(E_{e, N}\right)^{E}(x)$;

6. $\left(E_{e, N}\right)^{E}(x)=\left(E_{e, N}\right)^{E}\left(N^{E}(x)\right)$ for all $x \in L$;

Proof: First of all, notice that if $e$ is a fixed point of $N$, then $N^{E}(s(e))=$ $s(N(r(s(e))))=s(N(e))=s(e)$ what means that $s(e)$ is a fixed point of $N^{E}$. Taking this into account, it follows that:

1. $\left(E_{e, N}\right)^{E}(s(e))=s\left(E_{e, N}(r(s(e)))\right)=s\left(E_{e, N}(e)\right)=s\left(1_{M}\right)=1_{L} ;$ 
2. If $\left(E_{e, N}\right)^{E}(x)=1_{L}$ for a $x \in L$ then $s\left(E_{e, N}(r(x))\right)=1_{L}$ what implies $E_{e, N}(r(x))=1_{M}$ and hence by Definition 6.1 we have $r(x)=e$. Since $r$ is a lower retraction $s \circ r \leqslant i d_{L}$ then $s(e)=s(r(x)) \leqslant{ }_{L} x$.

3. Analogous to the previous item, considering that $i d_{L} \leqslant s \circ r$ if $r$ is an upper retraction.

4. Suppose $\left(E_{e, N}\right)^{E}(x)=0_{L}$ for a $x \in L$. Thus, $=s\left(E_{e, N}(r(x))\right)=0_{L}$ which means that $E_{e, N}(r(x))=0_{M}$ implying $r(x)=0_{M}$ or $r(x)=$ $1_{M}$. Hence $x=0_{L}$ or $x=1_{L}$. On the other hand, it is clear that $\left(E_{e, N}\right)^{E}\left(0_{L}\right)=\left(E_{e, N}\right)^{E}\left(1_{L}\right)=0_{L}$.

5. If $s(e) \leqslant_{L} x \leqslant_{L} y$ then $e \leqslant_{M} r(x) \leqslant_{M} r(y)$. In this case, we have that $\left(E_{e, N}\right)^{E}(y)=s\left(E_{e, N}(r(y))\right) \leqslant_{L} s\left(E_{e, N}(r(x))\right)=\left(E_{e, N}\right)^{E}(x)$ since $E_{e, N}(r(y)) \leqslant_{M} E_{e, N}(r(x))$. Analogously, one can prove the same thesis supposing $y \leqslant_{L} x \leqslant_{L} s(e)$.

6. For each $x \in L$ it follows

$$
\begin{aligned}
\left(E_{e, N}\right)^{E}\left(N^{E}(x)\right) & =s\left(E_{e, N}\left(r\left(N^{E}(x)\right)\right)\right) \\
& =s\left(E_{e, N}(r(s(N(r(x)))))\right) \\
& =s\left(E_{e, N}(N(r(x)))\right) \\
& =s\left(E_{e, N}(r(x))\right) \\
& =\left(E_{e, N}\right)^{E}(x)
\end{aligned}
$$

Remark 4. Proposition 6.2 shows again that the extension method via retraction is not efficient in extending normal $E_{e, N}$-functions, since property 1 of Definition 6.1 is not met by $\left(E_{e, N}\right)^{E}$, and just a weaker version holds (items 2 and 3 of Proposition 6.2), as observed for restricted equivalence functions and restricted dissimilarity functions.

Proposition 6.3. Let $M \unlhd L$ with respect to $\left(r_{1}, r_{2}, s\right), \odot: M \times M \rightarrow L$ be an e-operator and e be a fixed point of strong negation $N: M \rightarrow M$. If $E_{e, N}: M \rightarrow M$ is a normal $E_{e, N}$-function then its extension $\left(E_{e, N}\right)_{\odot}^{E}: L \rightarrow L$ given by

$$
\left(E_{e, N}\right)_{\odot}^{E}(x)=E_{e, N}\left(r_{1}(x)\right) \odot E_{e, N}\left(r_{2}(x)\right)
$$

for each $x \in L$, is a normal $E_{s(e), N_{\odot}^{E}}$-function.

Proof: It is clear that $s(e)$ is a fixed point of $N_{\odot}^{E}$ since $e$ is a fixed point of $N$. Moreover, 
1. if $\left(E_{e, N}\right)_{\odot}^{E}(x)=1_{L}$ then $E_{e, N}\left(r_{1}(x)\right) \odot E_{e, N}\left(r_{2}(x)\right)=1_{L}$ and by Lemma 4.15 we have $E_{e, N}\left(r_{1}(x)\right)=1_{M}$ and $E_{e, N}\left(r_{2}(x)\right)=1_{M}$ which means that $r_{1}(x)=e$ and $r_{2}(x)=e$. Hence $s(e)=s\left(r_{1}(x)\right) \leqslant_{L} x$ and $x \leqslant_{L}$ $s\left(r_{2}(x)\right)=s(e)$ allowing to conclude that $x=s(e)$. On the other hand,

$$
\begin{aligned}
\left(E_{e, N}\right)_{\odot}^{E}(s(e)) & =E_{e, N}\left(r_{1}(s(e))\right) \odot E_{e, N}\left(r_{2}(s(e))\right) \\
& =E_{e, N}(e) \odot E_{e, N}(e) \\
& =1_{M} \odot 1_{M}=s\left(1_{M}\right)=1_{L}
\end{aligned}
$$

Therefore, $\left(E_{e, N}\right)_{\odot}^{E}(x)=1_{L}$ if and only if $x=s(e)$.

2. Note that

$$
\begin{aligned}
\left(E_{e, N}\right)_{\odot}^{E}\left(0_{L}\right) & =E_{e, N}\left(r_{1}\left(0_{L}\right)\right) \odot E_{e, N}\left(r_{2}\left(0_{L}\right)\right) \\
& =E_{e, N}\left(0_{M}\right) \odot E_{e, N}\left(0_{M}\right) \\
& =0_{M} \odot 0_{M}=s\left(0_{M}\right)=0_{L}
\end{aligned}
$$

Similarly, one can verify that $\left(E_{e, N}\right)_{\odot}^{E}\left(1_{L}\right)=0_{L}$.

Now, suppose $\left(E_{e, N}\right)_{\odot}^{E}(x)=0_{L}$, i.e. $E_{e, N}\left(r_{1}(x)\right) \odot E_{e, N}\left(r_{2}(x)\right)=0_{L}$. Thus, $E_{e, N}\left(r_{1}(x)\right)=0_{M}$ and $E_{e, N}\left(r_{2}(x)\right)=0_{M}$ by Lemma 4.15 and hence either $r_{1}(x)=0_{M}$ or $r_{1}(x)=1_{M}$ or $r_{2}(x)=0_{M}$ or $r_{2}(x)=1_{M}$ which implies that $x=0_{L}$ or $x=1_{L}$.

3. Supposing $s(e) \leqslant_{L} x \leqslant_{L} y$ it is easy to see that $e \leqslant_{M} r_{1}(x) \leqslant_{M}$ $r_{1}(y)$ and $e \leqslant_{M} r_{2}(x) \leqslant_{M} r_{2}(y)$, then $E_{e, N}\left(r_{1}(y)\right) \leqslant_{M} E_{e, N}\left(r_{1}(x)\right)$ and $E_{e, N}\left(r_{2}(y)\right) \leqslant_{M} E_{e, N}\left(r_{2}(x)\right)$ and hence $E_{e, N}\left(r_{1}(y)\right) \odot E_{e, N}\left(r_{2}(y)\right) \leqslant_{L}$ $E_{e, N}\left(r_{1}(x)\right) \odot E_{e, N}\left(r_{2}(x)\right)$ since $\odot$ is isotonic. Therefore, $\left(E_{e, N}\right)_{\odot}^{E}(y)=$ $E_{e, N}\left(r_{1}(y)\right) \odot E_{e, N}\left(r_{2}(y)\right) \leqslant_{L} E_{e, N}\left(r_{1}(x)\right) \odot E_{e, N}\left(r_{2}(x)\right)=\left(E_{e, N}\right) \stackrel{E}{\odot}(x)$. Case $y \leqslant_{L} x \leqslant_{L} s(e)$ is analogous.

4. Finally, take $x \in L$. We know that $r_{1}(x) \leqslant_{M} r_{2}(x)$ and hence $N\left(r_{2}(x)\right) \leqslant \leqslant_{M}$ $N\left(r_{1}(x)\right)$ and by Lemma 4.11 we have that $r_{1}\left(N\left(r_{1}(x)\right) \odot N\left(r_{2}(x)\right)\right)=$ $N\left(r_{2}(x)\right)$ and $r_{2}\left(N\left(r_{1}(x)\right) \odot N\left(r_{2}(x)\right)\right)=N\left(r_{1}(x)\right)$. Therefore,

$$
\begin{aligned}
\left(E_{e, N}\right)_{\odot}^{E}\left(N_{\odot}^{E}(x)\right)= & E_{e, N}\left(r_{1}\left(N_{\odot}^{E}(x)\right)\right) \odot E_{e, N}\left(r_{2}\left(N_{\odot}^{E}(x)\right)\right. \\
= & \left(E_{e, N}\left(r_{1}\left(N\left(r_{1}(x)\right) \odot N\left(r_{2}(x)\right)\right)\right) \odot\right. \\
& \left(E_{e, N}\left(r_{2}\left(N\left(r_{1}(x)\right) \odot N\left(r_{2}(x)\right)\right)\right)\right) \\
= & E_{e, N}\left(N\left(r_{2}(x)\right)\right) \odot E_{e, N}\left(N\left(r_{1}(x)\right)\right) \\
= & E_{e, N}\left(N\left(r_{1}(x)\right)\right) \odot E_{e, N}\left(N\left(r_{2}(x)\right)\right) \\
= & \left(E_{e, N}\right)_{\odot}^{E}(x)
\end{aligned}
$$


Theorem 6.4. Let $N$ be a strong L-negation and e be a fixed point of $N$. If $R E F: L^{2} \rightarrow L$ is an $L-R E F$, then the function given by $E_{e, N}(x)=$ $R E F(x, N(x))$ for all $x \in L$ is a $E_{e, N}$-function.

\section{Proof:}

1. Suppose that $E_{e, N}(x)=1_{L}$. Thus, $\operatorname{REF}(x, N(x))=1_{L}$ and by $(L 2)$ we have $x=N(x)$. Then $x=e$. Reciprocally, if $x=e$ then $E_{e, N}(e)=$ $\operatorname{REF}(e, N(e))=\operatorname{REF}(e, e)=1_{L}$.

2. If $E_{e, N}(x)=0_{L}$, then $\operatorname{REF}(x, N(x))=0_{L}$. Hence either $x=1_{L}$ and $N(x)=0_{L}$ or $x=0_{L}$ and $N(x)=1_{L}$, i.e. $x=1_{L}$ or $x=0_{L}$. On the other hand, if $x=0_{L}$ then $E_{e, N}(x)=\operatorname{REF}\left(0_{L}, N\left(0_{L}\right)\right)=0_{L}$ and if $x=1_{L}$ then $E_{e, N}(x)=R E F\left(1_{L}, N\left(1_{L}\right)\right)=0_{L}$.

3. Note that if $e \leqslant_{L} x \leqslant_{L} y$ then $N(y) \leqslant_{L} N(x) \leqslant_{L} N(e)$. Since $N(e)=e$ we have that $N(y) \leqslant_{L} N(x) \leqslant_{L} x \leqslant_{L} y$ and hence $R E F(y, N(y)) \leqslant_{L}$ $R E F(x, N(x))$ by $(L 5)$. Thus $E_{e, N}(y) \leqslant_{L} E_{e, N}(x)$.

Now, suppose that $y \leqslant_{L} \quad x \leqslant_{L} e$. In this case, we have that $y \leqslant{ }_{L}$ $x \leqslant{ }_{L} N(x) \leqslant_{L} N(y)$ and again by $(L 5)$ it can be concluded that $R E F(y, N(y)) \leqslant_{L} R E F(x, N(x))$, i.e. $E_{e, N}(y) \leqslant_{L} E_{e, N}(x)$.

4. For all $x \in L$ we have

$$
\begin{aligned}
E_{e, N}(N(x)) & =\operatorname{REF}(N(x), N(N(x))) \\
& =\operatorname{REF}(N(x), x) \\
& =\operatorname{REF}(x, N(x)) \\
& =E_{e, N}(x)
\end{aligned}
$$

Corollary 6.5. Let $M \unlhd L$ with respect to $\left(r_{1}, r_{2}, s\right), \odot: M \times M \rightarrow L$ be an e-operator and REF : $M^{2} \rightarrow M$ a restricted equivalence function. If $e$ is a fixed point of a strong negation $N$ on $M$, then the function $E_{s(e), N_{\odot}^{E}}: L^{2} \rightarrow L$ given by $E_{s(e), N_{\odot}^{E}}(x)=R E F_{\odot}^{E}\left(x, N_{\odot}^{E}(x)\right)$ for all $x \in L$, is a normal $E_{s(e), N_{\odot}^{E}-}$ function.

Proof: Straightforward from Proposition 4.14 and Theorem 6.4. 


\section{Construction of similarity, distance and entropy measures}

Restricted equivalence functions and restricted dissimilarity functions on the unit interval have been used to define similarity measures and distance measures [8] between fuzzy sets.

In this section we define similarity measures and distance measures on general $L$-fuzzy sets giving some methods to construct them. In particular, restricted equivalence and restricted dissimilarity functions on a bounded lattice $L$ are applied.

Definition 7.1. [16] Let $L$ be a bounded lattice. An L-fuzzy set over the finite universe $U$ is a mapping $F: U \longrightarrow L$. We denote by $n \in \mathbb{N}$ the cardinal number of the universe $U$ and by $L F(U)$ to the set of all the $L$-fuzzy sets in the universe $U$.

In the development of this section we need the concept of aggregation function. Besides, the aggregation functions used in the constructions must fulfill some extra properties.

Definition 7.2. [25] Let $L$ be a bounded lattice. An aggregation function $A$ is a mapping $A: L^{n} \longrightarrow L$ satisfying the properties:

(A1) $A\left(0_{L}, \ldots, 0_{L}\right)=0_{L}, \quad A\left(1_{L}, \ldots, 1_{L}\right)=1_{L}$, and

(A2) $A\left(x_{1}, \ldots, x_{n}\right) \leqslant_{L} A\left(y_{1}, \ldots, y_{n}\right)$ whenever $x_{i} \leqslant_{L} y_{i}$ for all $i=\{1, \ldots, n\}$, namely, $A$ is monotonic increasing in all its arguments.

The properties we demand are:

(A3) $A\left(x_{1}, \ldots, x_{n}\right)=0_{L}$ if and only if $x_{i}=0_{L}$ for all $i \in\{1, \ldots, n\}$;

(A4) $A\left(x_{1}, \ldots, x_{n}\right)=1_{L}$ if and only if $x_{i}=1_{L}$ for all $i \in\{1, \ldots, n\}$.

Both $L$-REF and $L$-RDF can be used to compare two $L$-fuzzy sets. However, it is important to mention that there exist different axiomatic definitions of similarity measures on fuzzy sets (the lattice $L=[0,1]$ ). We base our definition on the one by Liu [22], but generating a slightly different notion of similarity measure on $L$-fuzzy sets. In the rest of this section $U=\left\{u_{1}, \ldots, u_{n}\right\}$. 
Definition 7.3. Let $L$ be a bounded lattice and $N$ a negation on $L$. A similarity measure on L-fuzzy sets is a mapping $S M: L F(U) \times L F(U) \longrightarrow L$ such that:

(SM1) $S M\left(F_{1}, F_{2}\right)=S M\left(F_{2}, F_{1}\right)$ for every $F_{1}, F_{2} \in L F(U)$.

(SM2) $S M\left(F_{1}, F_{2}\right)=0_{L}$ if and only if $\left\{F_{1}\left(u_{i}\right), F_{2}\left(u_{i}\right)\right\}=\left\{0_{L}, 1_{L}\right\}$ for all $u_{i} \in U$.

(SM3) $S M\left(F_{1}, F_{2}\right)=1_{L}$ if and only if $F_{1}\left(u_{i}\right)=F_{2}\left(u_{i}\right)$ for all $u_{i} \in U$.

(SM4) For all $F_{1}, F_{2}, F_{3} \in F(X)$, if $F_{1} \leq_{L F} F_{2} \leq_{L F} F_{3}$, then $S M\left(F_{1}, F_{3}\right) \leqslant{ }_{L}$ $S M\left(F_{1}, F_{2}\right)$ and $S M\left(F_{1}, F_{3}\right) \leqslant L S M\left(F_{2}, F_{3}\right)$.

Proposition 7.4. Let $L$ be a bounded lattice, and let $N$ be a strict negation on $L$. If $A: L^{n} \longrightarrow L$ is an aggregation function satisfying (A3) and (A4) and $R E F: L^{2} \longrightarrow L$ is an $L-R E F$ with respect to $N$, then the mapping $S M: L F(U) \times L F(U) \longrightarrow L$ given by:

$$
S M\left(F_{1}, F_{2}\right)=A\left(R E F\left(F_{1}\left(u_{1}\right), F_{2}\left(u_{1}\right)\right), \ldots, \operatorname{REF}\left(F_{1}\left(u_{n}\right), F_{2}\left(u_{n}\right)\right)\right)
$$

is a similarity measure.

Proof: Straightforward by Prop. 4 of [7].

Similar to the case of similarity measure, we base the generalization of distance measure in Liu's axiomatic definition [22].

Definition 7.5. Let $L$ be a bounded lattice and $N$ a negation on $L$. A distance measure on L-fuzzy sets is a mapping $D: L F(U) \times L F(U) \longrightarrow L$ such that:

(D1) $D\left(F_{1}, F_{2}\right)=D\left(F_{2}, F_{1}\right)$ for every $F_{1}, F_{2} \in L F(U)$.

(D2) $D\left(F_{1}, F_{2}\right)=0_{L}$ if and only if $F_{1}\left(u_{i}\right)=F_{2}\left(u_{i}\right)$ for all $u_{i} \in U$.

(D3) $D\left(F_{1}, F_{2}\right)=1_{L}$ if and only if $\left\{F_{1}\left(u_{i}\right), F_{2}\left(u_{i}\right)\right\}=\left\{0_{L}, 1_{L}\right\}$ for all $u_{i} \in U$.

(D4) For all $F_{1}, F_{2}, F_{3} \in F(X)$, if $F_{1} \leq_{L F} F_{2} \leq_{L F} F_{3}$, then $D\left(F_{1}, F_{2}\right) \leqslant_{L}$ $D\left(F_{1}, F_{3}\right)$ and $D\left(F_{2}, F_{3}\right) \leqslant{ }_{L} D\left(F_{1}, F_{3}\right)$.

Proposition 7.6. Let $L$ be a bounded lattice. If $A: L^{n} \longrightarrow L$ is an aggregation function satisfying (A3) and (A4) and $d_{L}: L^{2} \longrightarrow L$ is an $L-R D F$, then the mapping $D: L F(U) \times L F(U) \longrightarrow L$ given by:

$$
D\left(F_{1}, F_{2}\right)=A\left(d_{L}\left(F_{1}\left(u_{1}\right), F_{2}\left(u_{1}\right)\right), \ldots, d_{L}\left(F_{1}\left(u_{n}\right), F_{2}\left(u_{n}\right)\right)\right)
$$

is a distance measure. 
Proof: Straightforward by Prop. 4 of [8].

Finally, normal functions on the lattice $L$ are applied to construct entropy measures on $[0,1][8]$. Next, we define and generate entropy functions on a general lattice $L$ using $L$-normal $E_{e, N}$ functions.

Definition 7.7. Let $L$ be a bounded lattice and $N$ a negation on $L$. An entropy measure on L-fuzzy sets is a mapping $E: L F(U) \times L F(U) \longrightarrow L$ such that:

(E1) $E(F)=1_{L}$ if and only if $F(u)=e$ for all $u \in U$;

(E2) $E(F)=0_{L}$ if and only if $F(u)=0_{L}$ or $F(u)=1_{L}$ for all $u \in U$;

(E3) For all $F_{1}, F_{2} \in L F$ such that for all $u \in U$ either $e \leqslant_{L} F_{1}(u) \leqslant_{L} F_{2}(u)$ or $F_{2}(u) \leqslant_{L} F_{1}(u) \leqslant_{L} e$, it holds $\left.E_{(} F_{2}\right) \leqslant \leqslant_{L} E\left(F_{1}\right)$;

(E4) $E(F)=E(N(F))$ for all $F \in L F$, where $N(F)$ denotes the L-fuzzy set whose membership degree for each element $u \in U$ is $N(F(u))$.

Proposition 7.8. Let $L$ be a bounded lattice. If $A: L^{n} \longrightarrow L$ is an aggregation function satisfying (A3) and (A4) and $E_{e, N}$ is a normal function associated to $N$ (a strong negation with a fixed point $e$ ), then the mapping $E: L F(U) \times L F(U)$ given by

$$
E(F)=A\left(E_{e, N}\left(F\left(u_{1}\right)\right), \ldots, E_{e, N}\left(F\left(u_{n}\right)\right)\right)
$$

is an entropy measure.

Proof: Straightforward by Prop. 4 of [8].

Remark 5. Note that each color image is composed of several channels. For example, an RGB image is composed of three channels: red, green and blue. In this setting, each pixel of the image is associated with three values in the chain (bounded lattice $L=\{0,1, \ldots, 255\}$, i.e., the pixel $x_{i j}=\left(a_{i j}, b_{i j}, c_{i j}\right)$ where $a_{i j}, b_{i j}, c_{i j} \in L$.

Thus given $k \in \mathbb{N}^{+}$, the lattice of matrixes of $L^{k}$ with dimension $m \times n$ can be seen as the set of all images of $m \times n$ pixels with $k$ channels.

Consequently, the given constructions on this section can be applied to define the similarity, dissimilarity and entropy measures on color images.

Example 7.9. Due to the inherent uncertainty associated with the intensity of each pixel, the representation of an image by means of interval-valued 


\begin{tabular}{ccc}
$S M(A, B)$ & $S M(A, C)$ & $S M(A, D)$ \\
\hline$[0.9466,0.9735]$ & {$[0.8834,0.9374]$} & {$[0.8215,0.8998]$}
\end{tabular}

Table 2: Similarity measure between image A and images B, C and D

has been proposed in the literature [2, 19, 21, 24]. Based on this representation, each pixel is associated with an interval (a closed subinterval of the unit interval, as in Example 4.10). The interval associated with each pixel can be easily generated by taking the minimum intensity (of the original image) of a small area around the pixel as the lower bound and the maximum intensity as the upper bound. Considering the interval-valued images in Figure 2, which image $(B, C$ or $D)$ is more similar to image A? In order to answer this question, in this example we propose to use a similarity measure constructed by means of Proposition 7.5 taking as aggregation function $A\left(\mathbf{x}_{1}, \ldots, \mathbf{x}_{n}\right)=\left[\frac{1}{n} \sum_{i=1}^{n} \underline{x}_{i}, \frac{1}{n} \sum_{i=1}^{n} \bar{x}_{i}\right]$ and as restricted equivalence function the one used in Example 4.17. In this way, the similarity measure allows to stablish a comparison between images. The similarity obtained is shown in Table 2. Observe that, according to this similarity measure, $S M(A, D) \leq_{L} S M(A, C) \leq_{L} S M(A, B)$, which agrees with visual comparison of images.

\section{Final Remarks}

This work has addressed two different methods (via retractions EMR and via e-operators EMP) to extend restricted equivalence functions, restricted dissimilarity functions and $E_{e, N}$-normal functions.

As observed in previous works $[28,30,31]$, results have shown that the EMP method is more efficient in preserving properties of extended operators. We have noted that it is not possible to extend restricted equivalence functions by using EMR (just a weakened version of it, see Section 4.1). However, the extension by e-operators has worked successfully.

We have also studied a methodology to obtain information measures, such as similarities, distances and entropy measure for $L$-fuzzy sets. This methodology is based on the aggregation, with certain conditions, of restricted equivalence functions, restricted dissimilarities functions and $E_{e, N^{-}}$ normal functions, respectively.

We have illustrated the theoretical developments of this work with several 


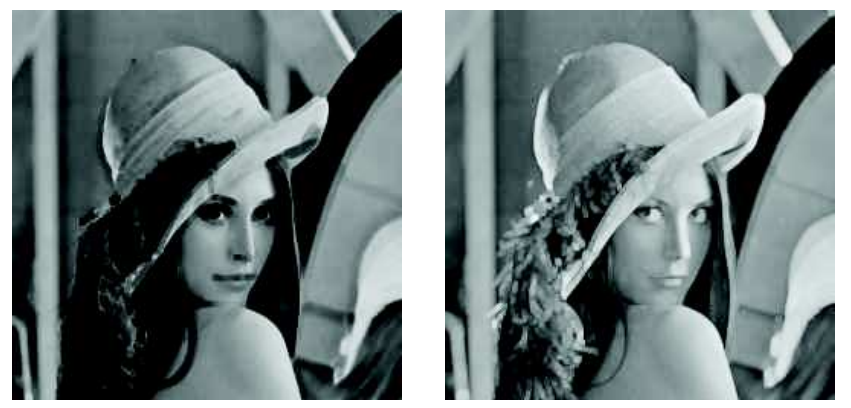

Interval-valued image A

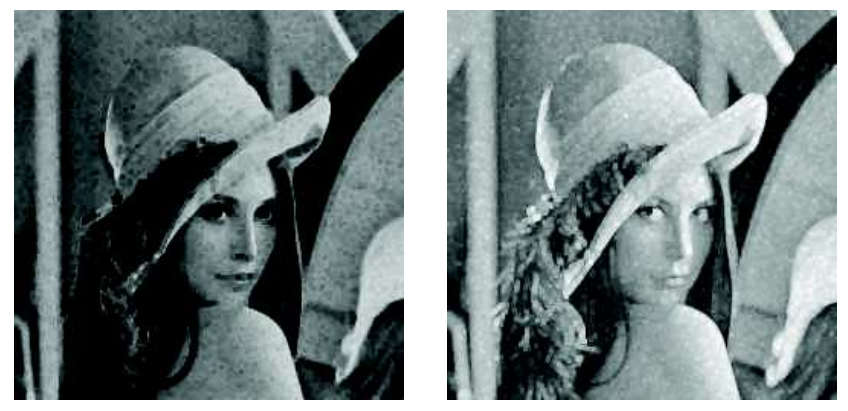

Interval-valued image B
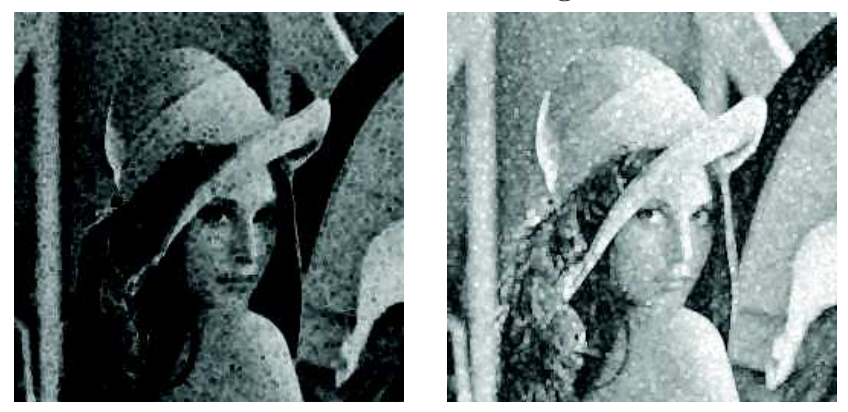

Interval-valued image $\mathrm{C}$
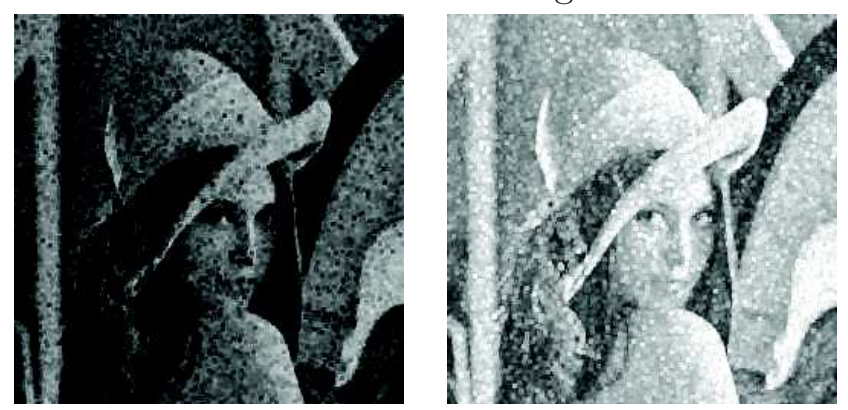

Interval-valued image D

Figure 2: Interval-valued images (lower bound on the left and upper bound on the right) 
examples where restricted equivalence functions have been extended to the interval setting. By aggregating interval restricted equivalence functions, we have obtained similarity measures that can be used to compare intervalvalued images, where the intensity of each pixel is represented by an interval.

For future works we intend to continue studying information measures defined on lattices, specially on those where there are elements which are not comparable. We also believe that some already defined concepts, for instance the length, might be useful for the interpretation of these measures.

\section{References}

[1] A.J. Baddeley. An error metric for images. Robust Computer Vision. Wichmann, Karlsruhe, pp. 59-78, 1992.

[2] E. Barrenechea, H. Bustince, B. De Baets and C. Lopez-Molina. Construction of interval-valued fuzzy relations with application to the generation of fuzzy edge images. IEEE Transactions on Fuzzy Systems, 19: 819-830, 2011.

[3] B. Bedregal. On interval fuzzy negations. Fuzzy Sets and Systems, 161: 2290-2313, 2010.

[4] B. Bedregal, G. Beliakov, H. Bustince, J. Fernandez, A. Pradera and R. Reiser. (S,N)-Implications on bounded lattices. M. Baczyński et al. (eds), Advances in Fuzzy Implication Functions. Studies in Fuzziness and Soft Computing, Springer, Berlin 2013.

[5] G. Beliakov, H. Bustince and D. Paternain. Image reduction using means on discrete product lattices. IEEE Transactions on Image Processing, 21(3):1080-1084, 2012.

[6] G. Birkhoff. Lattice theory. American Mathematical Society, Providence. RI, 1973.

[7] H. Bustince, E. Barrenechea and M. Pagola. Restricted equivalence functions. Fuzzy Sets and Systems, 157: 2333-2346, 2006.

[8] H. Bustince, E. Barrenechea and M. Pagola. Relationship between restricted dissimilarity functions, restricted equivalence functions and normal $E_{N}$-functions: Image thresholding invariant. Pattern Recognition Letters, 29: 525-536, 2008. 
[9] H. Bustince, E. Barrenechea, M. Pagola, J. Fernandez, Z. Xu, B. Bedregal, J. Montero, H. Hagras, F. Herrera and B. De Baets. A historical account of types of fuzzy sets and their relationships. IEEE Transactions on Fuzzy Systems, 24: 179-194, 2016.

[10] H. Bustince, J. Fernandez, R. Mesiar, A. Pradera and G. Beliakov. Restricted dissimilarity functions and penalty functions. EUSFLAT-LFA 2011, Aix-les-Bains, France, 2011.

[11] H. Bustince, V. Mohedano, E. Barrenechea and M. Pagola. Definition and construction of fuzzy DI-subsethood measures. Information Sciences, 176: 3190-3231, 2006.

[12] H. Bustince, M. Pagola and E. Barrenechea. Definition and construction of fuzzy DI-subsethood measures: Application to the global comparison of images. Information Science, 117(3): 906-929, 2007.

[13] S. Burris and H. P. Sankappanavar. A course in universal algebra. The Millennium Edition, New York 2005.

[14] B.A. Davey and H.A. Priestley. Introduction to lattices and order. Cambridge University Press, Cambridge, 2nd edition, 2002.

[15] J. Fodor and M. Roubens. Fuzzy preference modelling and multicriteria decision support. Kluwer Academic Publisher, Dordrecht, 1994.

[16] J. Goguen. L-fuzzy sets. Journal of Mathematical Analysis and Applications, 18(1):145-174, 1967.

[17] G. Grätzer. Lattice theory: First concepts and distributive lattices. Dover Publications, 2009.

[18] G. Grätzer. Lattice theory: Foundation. Birkhäuser, 2011.

[19] A. Jurio, M. Pagola, R. Mesiar, G. Beliakov and H. Bustince. Image magnification using interval information. IEEE Transactions on Image Processing, 20: 3112-2123, 2012.

[20] A. Kehagias and M. Konstantinidou. L-fuzzy valued inclusion measure, L-fuzzy similarity and L-fuzzy distance. Fuzzy Sets and Systems, 136(3):313-332, 2003. 
[21] L. Lima, B. Bedregal, H. Bustince, E. Barrenechea and M.P. da Rocha. An interval extension of homogeneous and pseudo-homogeneous t-norms and t-conorms. Information Sciences, 355-356:328-347, 2016.

[22] X. Liu. Entropy, distance measure and similarity measure of fuzzy sets and their relations Fuzzy Sets and Systems, 52(3):305-318,1992.

[23] R. Lowen. On fuzzy complements. Information Sciences, 14(2):107-113, 1978.

[24] A. Lyra, B. Bedregal, R. Callejas-Bedregal and A.D. Dória Neto. The interval digital images processing. WSEAS Transactions on Circuits and Systems, 3(2):229-233, 2004.

[25] R. Mesiar and M. Komorníková: Aggregation functions on bounded posets. In: Cornelis, C., Deschrijver, G., Nachtegael, M., Schockaert, S., Shi, Y. (eds.) 35 Years of Fuzzy Set Theory. STUDFUZZ, vol. 261, pp.3-17. Springer, Heidelberg (2010)

[26] M. Nachtegael, P. Sussner, T. Mélange, and E.E. Kerre. On the role of complete lattices in mathematical morphology: From tool to uncertianty model. Information Sciences, 181:1971-1988, 2011.

[27] E.S. Palmeira and B. Bedregal. Extension of fuzzy logic operators defined on bounded lattices via retractions. Computers and Mathematics with Applications, 63:1026-1038, 2012.

[28] E.S. Palmeira and B.C. Bedregal. Extension of t-subnorms on bounded lattices via retractions. 30th Annual Conference of the North American Fuzzy Information Processing Society. El Paso, TX, 2011.

[29] E.S. Palmeira, B. Bedregal and J.A.G. dos Santos Some results on extension of lattice-valued QL-implications. Journal of the Brazilian Computer Society 22(1): 4, 2016. DOI: 10.1186/s13173-016-0044-5.

[30] E.S. Palmeira, B. Bedregal and R. Mesiar. A new way to extend t-norms, t-conorms and negations. Fuzzy Sets and Systems, 240: 1-21, 2014.

[31] E.S. Palmeira, B. Bedregal, J. Fernandez and A. Jurio. On extension of lattice-valued implications via retractions. Fuzzy Sets and Systems, 240:66-85, 2014. 
[32] D. Paternain, G. Ochoa, I. Lizasoain, H. Bustince and R. Mesiar. Quantitative orness for lattice OWA operators. Information Fusion, 30: 2735, 2016.

[33] R.H.S Reiser, G.P. Dimuro, B. Bedregal, H.S. Santos and R. CallejasBedregal. S-implications on complete lattices and the interval constructor. TEMA: Tendências em Matemática Aplicada e Computacional, 9(1):143-154, 2008.

[34] C. Ronse. Why mathematical morphology needs complete lattices. Signal Processing, 21: 129-154,1990.

[35] S. Saminger-Platz, E. P. Klement and R. Mesiar. On extensions of triangular norms on bounded lattices. Indagationes Mathematicae, 19(1): 135-150, 2008.

[36] J. Sanz, A. Fernández, H. Bustince and F. Herrera. IVTURS: A linguistic fuzzy rule-based classification system based on a new interval-valued fuzzy reasoning method with tuning and rule selection. IEEE Transactions on Fuzzy Systems, 21:399-411, 2013.

[37] E. Trillas. Sobre funciones de negación en la teoria de conjuntos difusos. Stochastica, 3: 47-59, 1979. 